\title{
3D QSAR Studies of Coumarin Derivatives for Modifying the Pharmachophoric Sites Using Betti's Protocol
}

\author{
Kiran M. Khandarkar ${ }^{1,2, *}$, Manoj Shanti ${ }^{1}$, Mudrika Ahmed ${ }^{3}$, Jyotsna S. Meshram ${ }^{1,4}$ \\ ${ }^{1}$ Chemistry Department, Rashtrasant Tukadoji Maharaj Nagpur University, Nagpur, 440033, Maharashtra, India \\ ${ }^{2}$ G. H. Raisoni Academy of Engineering \& Technology, Nagpur, 440016, Maharashtra, India \\ ${ }^{3}$ Govt. Polytechnique College of Engineering, Sakoli, Bhandar, 441802, Maharashtra, India \\ ${ }^{4}$ School of Chemical Sciences, North Maharashtra University, Jalgaon,425001 Maharashtra, India \\ *Corresponding Author: kirankhandarkar@gmail.com
}

Copyright (C) 2013 Horizon Research Publishing All rights reserved.

\begin{abstract}
An effortless and straight forward approach to new substituted coumarin derivatives via Betti's condensation reaction of quinoline and chromone based aldehydes has been demonstrated successfully under ambient reaction conditions. Physico-chemical parameters, toxicity profiles, drug likeness were studied using various bioinformatical tools like Petra, Osiris and Molinspiration. Good correlations were found between the predicted binding free energies and the experimental inhibitory activities which suggested that the identified binding conformations of these potential inhibitors are reliable. These results viz. the good correlations between the inhibitory activities and the computational values, makes the molecules available for future protein-ligand interaction studies. It further provided useful information in understanding the structural and chemical features of the drug in designing and finding new potential inhibitors.
\end{abstract}

Keywords Drug likeness, Inhibitory activities, Betti's reaction, Coumarin

\section{Introduction}

Coumarins, an old class of compounds, are naturally occurring benzopyrene derivatives [1-5]. A lot of coumarins have been identified from natural sources, especially green plants. The pharmacological and biochemical properties and therapeutic applications of simple coumarins depend upon the pattern of substitution. Coumarins have attracted intense interest in recent years because of their diverse pharmacological properties [6, 7]. Among these properties, their cytotoxic effects were most extensively examined $[8,9]$. In this present work, we have tried to correlate the various physico-chemical parameters studied by chem-bioinformatics data generator engines with in vitro studies.

We now report a new synthetic approach to coumarin derivatives bearing chromone and quinoline side chain utilizing basic coumarin skeleton following Betti's protocol which is found to be new, one-pot, facile, and convenient methodology. Betti's derivatives are easily prepared by the condensation of 2-napthol, benzaldehyde and ammonia for 4-5 days in ethanol as reported [10]. But, in this approach, we have used 7-hydroxycoumarin in place of 2-napthol to produce the promising derivatives. Firstly, the coumarin skeleton has been constructed by literature method [11]. The coumarin skeleton thus obtained was employed for the generation of Betti's condensed product at room temperature isolated as well shining crystalline molecules bearing chromone and quinoline moieties attached to the core coumarin skeleton.

Coumarins, chromones and quinolines form a significant class of compounds in medicinal and pharmaceutical chemistry with several biological applications that include antibacterial [12, 13], antifungal [14-17] and antitumor activity $[18,19]$. They can be studied as a class of ligand for docking studies. Thus, coumarins containing chromone and quinoline centre are expected to have enhanced biological activities owing to the presence of multiple pharmacophores associated with the coupling of coumarin and chromone and quinoline in a single active ingredient.

To develop robust prediction models for the heterocycles bacterial inhibitory properties, solubility, bioavailability, drug likeness, toxicity study, etc. were calculated using various online physico-chemical properties predictor engines which give a design of the right molecule for the right target in a goal to improve bioselectivity. The 3D QSAR based modification of the pharmacophoes and the sites are supposed to enhance its bioactivity and add to the rational drug design.

\section{Materials and Methods}

\subsection{General}

The solvents and reagents used in the synthetic work were 
of analytical grade obtained from Qualigens India and were purified by distillation or crystallization where necessary and their boiling or melting points were compared with the available literature values. Melting points were determined in open capillaries and are uncorrected. ${ }^{1} \mathrm{HNMR}$ spectra were recorded on a Cryo-magnet Spectrometer $400 \mathrm{MHz}$ (Bruker) instrument using tetramethylsilane (TMS) as an internal standard and DMSO-d6 as a solvent at room temperature $\left({ }^{13} \mathrm{CNMR}\right.$ at $\left.75 \mathrm{MHz}\right)$. Chemical shifts are given in parts per million (ppm). Infrared spectra were recorded on Shimadzu-IR Prestige 21. Mass spectra were recorded on a Waters Micromass Q-T of Micro spectrometer. The reactions were monitored and the purity of products was checked out on pre-coated TLC plates (Silica gel 60 F254, Merck), visualizing the spots under ultraviolet light and iodine chamber.

\subsection{Biological Screening}

The agar cup plate method using Hi-Media agar medium was employed to study the antibacterial activity of (4a) and (4b) against Staphylococcus aureus, Proteus vulgaris, Pseudomonas aeruginosa, S. pyogenes, Klebsiella spp., Salmonella typhi and Escherichia coli. Preparation of nutrient broth, subculture, base layer medium, agar medium and peptone water was done as per the standard procedure. Each test compound $(50 \mathrm{mg})$ was dissolved in dimethylformamide $(50 \mathrm{~mL}, 1000 \mathrm{mg} / \mathrm{mL})$, which was used as sample solution. Sample size for all the compounds was fixed at $0.1 \mathrm{~mL}$. Using a sterilized cork borer, cups were scooped out of Agar medium contained in a petri dish which was previously inoculated with the microorganisms. The test compound solution $(0.1 \mathrm{~mL})$ was applied to the wells with the help of a micropippete and the Petri dishes were subsequently incubated at $37^{\circ} \mathrm{C}$ for $24 \mathrm{hrs}$ and then extended for next $24 \mathrm{hrs}$. Ampicillin and Streptomycin were used as reference drugs and dimethylformamide as a negative control. Zones of inhibition produced by each compound were measured in millimetres, and the results are listed in (Table 2).

\section{3. Minimum Inhibitory Concentration (MIC)}

MIC is defined as the lowest compound concentration preventing visible bacterial growth. Minimum inhibitory concentrations of the compounds were determined by taking different concentrations of the compound in DMF. The MICs were determined using the Agar cup plate method by preparing dilutions of $10,25,50,75$ and $100 \mathrm{mg} / \mathrm{mL}$ of the compounds with both the compounds (4a) and (4b) of which (4a) is having least MIC in general. It was observed that the MIC of selected compounds was in the range of $25-100 \mathrm{mg} / \mathrm{mL}$. The results obtained are tabulated in Tables $3 \mathrm{~A}$ and $\mathrm{B}$.

\subsection{Synthesis}

\subsubsection{General Procedure (Compounds $4 \mathrm{a} \& 4 \mathrm{~b})$ :}

In a typical experiment, Coumarin (3) $1.0 \mathrm{~g}(6.5 \mathrm{mmol})$ was dissolved in $10 \mathrm{ml}$ of $95 \%$ of ethanol. To this solution aldehyde (chromonaldehyde (5)/2-chloroquinonaldehyde (6)) $(6.5 \mathrm{mmol})$ was added with stirring. After $5 \mathrm{~min} 0.94 \mathrm{~g}$ $(6.5 \mathrm{mmol})$ of benzocaine (4) was dissolved in $10 \mathrm{ml}$ of $95 \%$ ethanol at room temperature and added to the previous mixture. The solution was turned to a brown colour. The system was sealed and allowed to stir for $3 \mathrm{hrs}$ and then kept undisturbed at room temperature for another $12 \mathrm{hrs}$. After about $12 \mathrm{~h}$ of standing of reaction mixture at RT, the condensed product was separated as pale needles. It was isolated by filtration on the suction pump and washed well with cold ethanol. Analytically pure product was obtained by recrystallization by means of ethanol. The second crop of the product was obtained by allowing the mother liquor to stand undisturbed for net 24 hours at ambient temperature conditions.

The structures of compounds (4a) and (4b) were deduced from their elemental analyses and their IR, ${ }^{1} \mathrm{H},{ }^{13} \mathrm{C}$ NMR and mass spectral data. All the compounds were obtained in excellent yield as crystalline solids.

Ethyl4-\{[(7-hydroxy-4-methyl-2-oxo-2H-chromen-8-yl)( 4-oxo-4H-chromen-2-yl) methyl]amino benzoate (4a): Pale green crystalline solid, yield $90 \%$ (4.477 g, crystallization) or $97 \%\left(4.825 \mathrm{~g}\right.$, filtration), m. p. $140-142{ }^{\circ} \mathrm{C}$; refractive index nD22: 1.679. Anal. Calcd. for C29H23NO7: C, 70.01; H, 4.66; N, $2.82 \%$. Found: C, 70.11; H, 4.73; N, $2.76 \%$. IR (cm-1) v: $3457.7(\mathrm{OH}), 1714.6$ (cyclic CO), $3436.3(\mathrm{NH})$, 1358.3 (C-N stretching), 1735.7 (COOC2 H5). ${ }^{1} \mathrm{HNMR}$ (DMSO, $400 \mathrm{MHz}, \mathrm{ppm}, \mathrm{J} \mathrm{Hz}) \delta \mathrm{H}: 11.81(\mathrm{~s}, 1 \mathrm{H}$, coumarin-OH); 1.33 (s, 3H, methyl coumarin); 7.09-7.95 (m, $3 \mathrm{H}$, coumarin- $\mathrm{H}) ; 5.98(\mathrm{~s}, 1 \mathrm{H}$, methine); $3.82(\mathrm{~s}, 1 \mathrm{H} \mathrm{sec}$. amine $\mathrm{HN}-)$; 7.56-7.98 (m, 4H, Ar-H ); $1.10(\mathrm{t}, 3 \mathrm{H},-\mathrm{CH} 3, \mathrm{~J}=$ $8.0) ; 4.33$ (q, $2 \mathrm{H},-\mathrm{OCH} 2, \mathrm{~J}=4.0,8.0) ; 7.14-8.18$ (m, $5 \mathrm{H}$, Chromon-H). ${ }^{13} \mathrm{C}$ NMR $(\mathrm{CDCl} 3,75 \mathrm{MHz}, \mathrm{ppm}) \delta \mathrm{C}: 14.6$, $19.2,53.6,61.2,110.1,111.9,112.1,112.4,112.6,116.3$, $118.6,122.5,123.4,124.2,124.6,125.9,130.8,135.2,149.8$, $150.3,152.6,153.6,157.4,161.6,166.3,167.3, \quad 178.2$.MS, m/z: $497(\mathrm{M}+, 100 \%)$.

Ethyl4-\{[(2-chloroquinolin-3-yl)(7-hydroxy-4-methyl-2oxo-2H-chromen-8-yl)methyl] amino benzoate (4b): Bright yellow amorphous solid, yield $96 \%$ (4.943 g, crystallization) or $97 \%(5.0 \mathrm{~g}$, filtration $)$, m. p. $98-101^{\circ} \mathrm{C}$; refractive index nD22: 1.691. Anal. Calcd. for C29H23ClN2O5: C, 67.64; H, $4.50 ; \mathrm{Cl}, 6.88 ; \mathrm{N}, 5.44 \%$. Found: $\mathrm{C}, 67.72 ; \mathrm{H}, 4.58 ; \mathrm{Cl}, 6.92$; $\mathrm{N}, 5.38 \%$. IR (cm-1) v: $3498.3(\mathrm{OH}), 1715.6$ (cyclic CO), 3419.9 (NH), 1277.7 (C-N stretching), 1732.4 (COOC2 H5), 1098.6 (Cl). ${ }^{1} \mathrm{HNMR}$ (DMSO, $400 \mathrm{MHz}, \mathrm{ppm}, \mathrm{J} \mathrm{Hz}$ ) $\delta \mathrm{H}$ : $10.53(\mathrm{~s}, 1 \mathrm{H}$, coumarin-OH); 2.37 (s, 3H, methyl coumarin); 6.55-7.46 (m, 3H, coumarin- $\mathrm{H}) ; 6.80(\mathrm{~s}, 1 \mathrm{H}$, sec. amine $-\mathrm{NH}) ; 6.71-7.61(\mathrm{~m}, 4 \mathrm{H}, \mathrm{Ar}-\mathrm{H}) ; 1.33$ ( t, 3H, $-\mathrm{CH} 3, \mathrm{~J}=8.0$ ); $4.32(\mathrm{q}, 2 \mathrm{H},-\mathrm{CH} 2, \mathrm{~J}=4.0,8.0) ; 6.14(\mathrm{~s}, 1 \mathrm{H}$, methine); 7.74-9.22 (m, 5H, quinoline-H). ${ }^{13} \mathrm{C}$ NMR (CDCl3,75 MHz, ppm) $\delta \mathrm{C}: 13.8,20.1,45.8,61.2,112.0,112.2,112.5,112.7$, 118.6, 122.6, 124.4, 126.7, 127.0, 127.5, 130.1, 130.7, 131.0, 
136.2, 142.4, 147.4, 150.2, 152.4, 153.6, 155.1, 161.6, 166.7. MS, m/z: $514(\mathrm{M}+, 100 \%)$.

\section{Results and Discussion}

\subsection{Chemistry}

The target compounds were synthesized by a two-step synthetic protocol. In the first step, starting material 4-methyl-7-hydroxycoumarin (3) was prepared by the condensation reaction between 1 equiv resorcinol (1) and 1 equiv of ethylacetoacetate (2) as per the reported method (Scheme 1) [11].

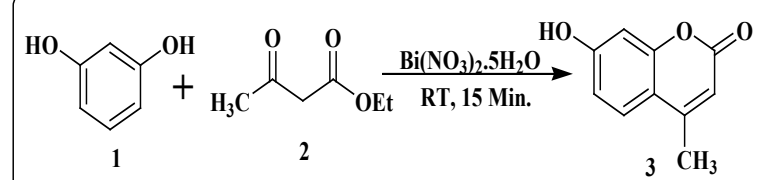

Scheme 1. Representation of synthetic methodology for 4-methyl-7-hydroxycoumarin

The product was isolated with $95 \%$ yield. The uncorrected mp was $179-181{ }^{\circ} \mathrm{C}$ and this closely matched the literature value of $180-182^{\circ} \mathrm{C}$. The synthesized coumarin (3) leaded in the second step of preparation; Betti's methodology was applied for the synthesis, which is a condensation reaction between phenol, benzaldehyde and amine in one-pot synthesis (Scheme 2) [10]. But in this present approach an aromatic amine has been introduced as the third component in place of ammonia. (Scheme 3) [20].

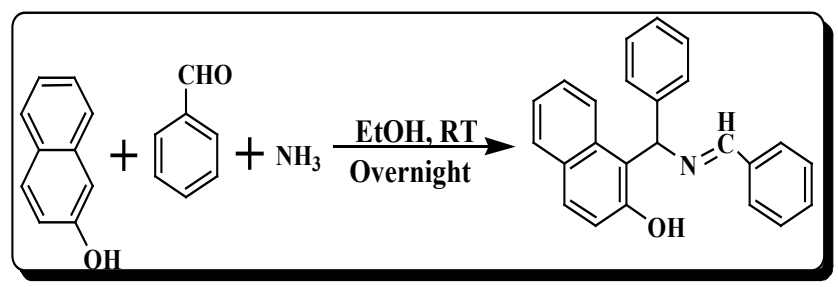

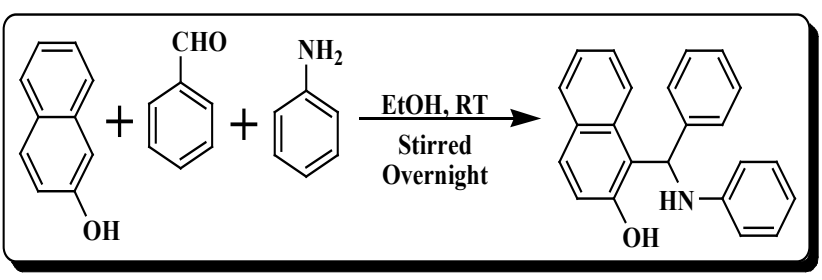

Scheme 3. Modified model representation of Betti's Method

In an approach, one equiv of coumarin (3), one equiv chromone (5)/quinoline (6) aldehydes and one equiv benzocaine (4) in $95 \%$ of alcohol were stirred for 3 hours and kept at room temperature for $12 \mathrm{~h}$. TLC checked reaction progress duly in a duration of 15 minutes. The products were isolated by simple and usual work up with $85-90 \%$ of yield economy. Thus, this is a $3-\mathrm{MCR}$ reaction of coumarin, chromone/quinoline aldehydes and benzocaine. It is the special case of Mannich reaction which consists of an amino alkylation of an acidic proton placed next to a carbonyl functional group with aldehydes and any amine. The executed synthetic strategy is highlighted in Scheme 4.

\subsection{In Vitro Antimicrobial Screening}

The in-vitro studies for (4a) and (4b) have been carried out against some pathogenic strains of bacteria. The Agar cup plate method was employed to determine the antibacterial activity of these agents, with Ampicillin and Streptomycin as the reference antibiotics. The synthesized compounds were examined against pathogenic strains of negative bacteria. The test results, presented in Table 1, suggest that compounds that both $4 \mathrm{a}$ and $4 \mathrm{~b}$ are highly active against almost all strains bacteria showing a broad spectrum of antibacterial activity particularly against Gram +ve bacteria. The minimum inhibitory concentrations were also found to be surprisingly matching with the standards as cited in the Table 2.

Scheme 2. General model representation of Betti's method

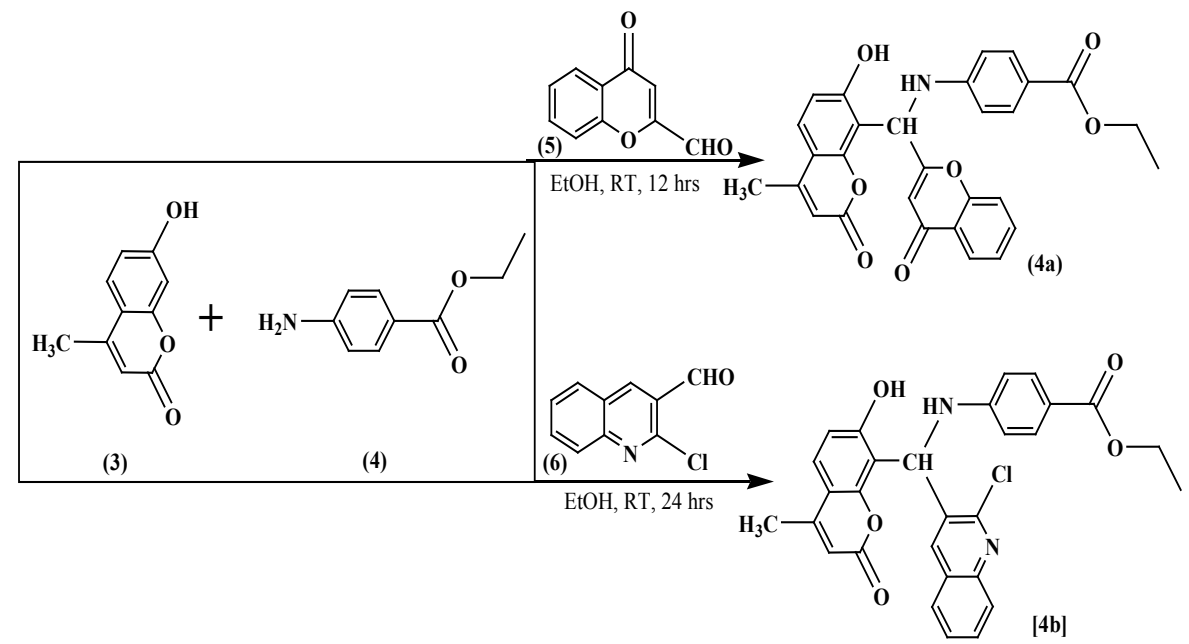

Scheme 4. Synthetic protocol for coumarin based Betti's bases. 
Table 1. Antibacterial activity of compounds

\begin{tabular}{|c|c|c|c|c|c|c|c|}
\hline \multirow[b]{2}{*}{ Comp. } & \multicolumn{7}{|c|}{ Bacterial Strains } \\
\hline & S. aureus & P. vulgaris & $\begin{array}{c}\text { S. } \\
\text { pyogenes }\end{array}$ & $\begin{array}{c}\text { Ps. } \\
\text { aeruginosa }\end{array}$ & Klebsiella spp. & $\begin{array}{c}\text { Salmonella } \\
\text { typhi }\end{array}$ & E. coli \\
\hline $4 a$ & +++ & + & ++ & +++ & +++ & ++ & + \\
\hline $4 b$ & +++ & ++ & +++ & ++ & ++ & +++ & ++ \\
\hline AMP & +++ & ++ & +++ & +++ & +++ & +++ & ++ \\
\hline STRE & +++ & +++ & +++ & +++ & +++ & +++ & +++ \\
\hline
\end{tabular}

$\mathrm{AMP}=$ Ampicillin, $\mathrm{STRE}=$ Streptomycin, Inactive $=-($ inhibition zone $<5 \mathrm{~mm})$; slightly active $=+($ inhibition zone $5-10 \mathrm{~mm}) ;$ moderately active $=$ $++($ inhibition zone $10-15 \mathrm{~mm})$; highly active $=+++($ inhibition zone $>15 \mathrm{~mm})$.

Table 2. Minimum inhibitory concentrations of compounds

\begin{tabular}{|c|c|c|c|c|c|c|c|}
\hline \multirow[b]{2}{*}{ Comp. } & \multicolumn{7}{|c|}{ Bacterial Strains } \\
\hline & S. aureus & P. vulgaris & S. pyogenes & Ps. aeruginosa & $\begin{array}{c}\text { Klebsiella } \\
\text { spp. }\end{array}$ & $\begin{array}{c}\text { Salmonella } \\
\text { typhi }\end{array}$ & E. coli \\
\hline $4 \mathrm{a}$ & $<25$ & $<100$ & $<50$ & $<25$ & $<25$ & $<50$ & $<100$ \\
\hline $4 b$ & $<25$ & $<100$ & $<50$ & $<50$ & $<50$ & $<25$ & $<50$ \\
\hline AMP & $<10$ & $<25$ & $<25$ & $<10$ & $<25$ & $<10$ & $<25$ \\
\hline STRE & $<10$ & $<10$ & $<25$ & $<25$ & $<10$ & $<25$ & $<10$ \\
\hline
\end{tabular}

AMP: Ampicillin, STRE: Streptomycin, Concentration in $\mu \mathrm{g} / \mathrm{mL}$.

Table 3. Molinspiration calculations of compounds (4a) and (4b)

\begin{tabular}{|c|c|c|c|c|c|c|c|c|}
\hline Compd & MW & mi $\log \mathrm{P}$ & TPSA & $\mathrm{OH}-\mathrm{NH}$ & Bond Rot. & $\begin{array}{c}\mathrm{n} \\
\text { viola- } \\
\text { tion }\end{array}$ & Vol. & $\begin{array}{l}\mathrm{O}- \\
\mathrm{N}\end{array}$ \\
\hline $4 a$ & 497 & 5.983 & 118.982 & 2 & 7 & 1 & 431 & 8 \\
\hline $4 b$ & 514 & 6.816 & 101.663 & 2 & 7 & 2 & 440 & 7 \\
\hline AMP & 349 & -0.87 & 113 & 4 & 4 & 0 & 299 & 7 \\
\hline STRE & 581 & -5.35 & 336 & 16 & 9 & 3 & 497 & 19 \\
\hline
\end{tabular}

\subsection{Virtual Screenings and Molecular Properties Calculations}

\subsubsection{Molinspiration Calculations}

MiLogP or cLogP (octanol/water partition coefficient) which is used to estimate solubility is calculated by the methodology developed by Molinspiration as a sum of fragment based contributions and correction factors (Tables 3 and 4). The method is very robust and is able to process practically all organic and most organometallic molecules $[21,22]$. Molecular Polar Surface Area TPSA is calculated based on the methodology published by Ertl et al. as a sum of fragment contributions [23]. O- and $\mathrm{N}$ - centered polar fragments are considered. PSA has been shown to be a very good descriptor characterizing drug absorption, including intestinal absorption, bioavailability, Caco- 2 permeability and blood brain barrier penetration. Prediction results of compounds (4a) and (4b) molecular properties (TPSA, GPCR ligand and ICM) are valued (Tables 4 and 6). lipophilicity ( $\log \mathrm{P}$ value) and polar surface area (PSA) values are two important properties for the prediction of per oral bioavailability of drug molecules [24]. Therefore we have calculated $\log$ P and PSA values for compounds (4a) and (4b) using molinspiration software programs and compared them with the values obtained for standard drugs Ampicillin and Streptomycin. For all the compounds, the calculated $\log \mathrm{P}$ values were around 5-6, but 5 is the upper limit for the drugs to be able to penetrate through biomembranes according to Lipinski's rules. The highest degree of lipophilicity was found with all the compounds which are an indication for good lipid solubility that will help the drug to interact with the membranes. The polar surface area (PSA) is calculated from the surface areas that are occupied by oxygen and nitrogen atoms and by hydrogen atoms attached to them. Thus, the PSA is closely related to the hydrogen bonding potential of a compound [25]. Molecules with PSA values of $140 \AA \AA$ or more are expected to exhibit poor intestinal absorption. Table 4 shows that all the compounds are within this limit. It has to be kept in mind that $\log$ P and PSA values are only two important, although not sufficient, criteria for predicting oral absorption of a drug [26]. To support this contention, it is to be noted that all the compounds have only one violation of the Rule of 5 .

Two or more violations of the Rule of 5 suggest the probability of problems in bioavailability [27]. All the compounds have only one violation of the Rule but that to with a small difference. Drug likeness of compounds is tabulated in Table 4. Drug likeness may be defined as a 
complex balance of various molecular properties and structure features which determine whether particular molecule is similar to the known drugs. These properties, mainly hydrophobicity, electronic distribution, hydrogen bonding characteristics, molecule size and flexibility and presence of various pharmacophores features influence the behavior of molecule in a living organism, including bioavailability, transport properties, affinity to proteins, reactivity, toxicity, metabolic stability and many others. Activity of all compounds and standard drugs were rigorously analyzed under four criteria of known successful drug activity in the areas of GPCR ligand activity, ion channel modulation, kinase inhibition activity, and nuclear receptor ligand activity.

Results are shown for the compounds in Table 4 by means of numerical assignment. Likewise all compounds have consistent negative values in all categories and numerical values conforming and comparable to that of standard drugs used for comparison. Therefore it is readily seen that all the compounds are expected to have near similar activity to standard drugs used based upon these four rigorous criteria (GPCR ligand, ion channel modulator, kinase inhibitor, and nuclear receptor ligand).

Table 4. Drug likeness of compounds.

\begin{tabular}{ccccccc}
\hline Compd & GPCR & ICM & KI & NRL & PI & EI \\
\hline 4a & -0.48 & -0.77 & -0.43 & -0.18 & -0.51 & -0.24 \\
4b & -0.36 & -0.62 & -0.27 & -0.18 & -0.45 & -0.17 \\
AMP & 0.04 & -0.47 & -0.71 & -0.61 & 0.87 & 0.25 \\
STREP & 0.09 & -0.16 & -0.17 & -0.18 & 0.65 & 0.38 \\
\hline
\end{tabular}

\subsubsection{Osiris Calculations}

Structure based design is now fairly routine but many potential drugs fail to reach the clinic because of ADME-Tox liabilities. One very important class of enzymes, responsible for many ADMET problems, is the cytochromes P450. Inhibition of these or production of unwanted metabolites can result in many adverse drug reactions. With our recent work on the drug design by combination of various pharmacophore sites by using heterocyclic structure, it is now possible to predict activity and/or inhibition with increasing success in two targets (bacteria and HIV) [21, 28]. This was done using a combined electronic/structure docking procedure and an example will be given here. The remarkably well behaved mutagenicity of diverse synthetic molecules classified in data base of CELERON Company of Swiss can be used to quantify the role played by various organic groups in promoting or interfering with the way a drug can associate with DNA. The Osiris calculations are tabulated in Tables 5.Toxicity risks (mutagenicity, tumorigenicity, irritation, reproduction) and physico-chemical properties (mi $\log \mathrm{P}$, solubility, drug likeness and drug score) of compounds (4a) and (4b) are calculated by the methodology developed by Osiris. The toxicity risk predictor locates fragments within a molecule, which indicate a potential toxicity risk. Toxicity risk alerts are an indication that the drawn structure may be harmful concerning the risk category specified. From the data evaluated in Table 5. indicates that all structures are supposed to be non-mutagenic, non-irritating with no reproductive effects when run through the mutagenicity assessment system shown with green spheres comparable with standard drugs used. The $\log \mathrm{P}$ value of a compound, which is the logarithm of its partition coefficient between n-octanol and water, is a well-established measure of the compound's hydrophilicity. Low hydrophilicities and therefore high $\log \mathrm{P}$ values may cause poor absorption or permeation. It has been shown for compounds to have a reasonable probability of being well absorb their $\log \mathrm{P}$ value must not be greater than 5. Along with this all compounds have shown good antibacterial screening results, are having low $\log \mathrm{P}$.

Table 5. Osiris calculations of compounds

\begin{tabular}{|c|c|c|c|c|c|c|c|c|c|}
\hline \multirow{2}{*}{ Compd. } & \multicolumn{4}{|c|}{ Predicted toxicity risks } & \multicolumn{5}{|c|}{ Molecular properties' calculations } \\
\hline & MUT & TUMO & IRRI & REP & MW & CLP & $\log S$ & DL & D-S \\
\hline $4 a$ & O & O & O & O & 497 & 4.65 & -5.63 & -7.46 & 0.20 \\
\hline $4 \mathrm{~b}$ & O & O & O & O & 514 & 5.67 & -6.09 & -8.74 & 0.15 \\
\hline AMP & O & O & O & O & 349 & -0.04 & -1.57 & 10.72 & 0.91 \\
\hline STREP & O & O & O & 0 & 581 & -0.96 & -0.96 & 0.83 & 0.43 \\
\hline
\end{tabular}

AMP: Ampicillin; STREP: Streptomycin; MUT: Mutagenic; TUMO: tumorigenic; IRRI: Irritant; REP: Reproductive effective. 
The aqueous solubility of a compound significantly affects its absorption and distribution characteristics. Typically, a low solubility goes along with a bad absorption and therefore the general aim is to avoid poorly soluble compounds. Our estimated $\log \mathrm{S}$ value is a unit stripped logarithm (base 10) of a compound's solubility measured in $\mathrm{mol} /$ liter. There are more than $80 \%$ of the drugs on the market have an (estimated) $\log \mathrm{S}$ value greater than -4 . Further, Table 5 shows drug likeness of compounds (4a) and (4b) which is in the comparable zone with that of standard drugs used for comparison. We have calculated overall drug score (DS) for compounds (4a) and (4b) and compared with that of standard drugs Ampicillin and Streptomycin used as shown in Table 5. The drug score combines drug likeness, mi $\log \mathrm{P}, \log \mathrm{S}$, molecular weight and toxicity risks in one handy value that may be used to judge the compound's overall potential to qualify for a drug. This value is calculated by multiplying contributions of the individual properties with the equation (1):

$$
\mathrm{DS}=\prod(1 / 2+1 / 2 \mathrm{Si}) \prod \mathrm{ti}
$$

Where, $S=1 / 1+\mathrm{e}^{\mathrm{ap}+\mathrm{b}}$

DS is the drug score. $\mathrm{Si}$ is the contributions calculated directly from of mi $\log \mathrm{P} ; \log \mathrm{S}$, molecular weight and drug likeness (pi) via the second equation, which describes a spline curve. Parameters a and $b$ are $(1,-5),(1,5),(0.012,-6)$ and $(1,0)$ for mi $\log \mathrm{P}, \log \mathrm{S}$, molecular weight and drug likeness, respectively. ti is the contributions taken from the four toxicity risk types. The ti values are 1.0, 0.8 and 0.6 for no risk, medium risk and high risk, respectively. The reported compounds (4a) and (4b) showed moderate to good drug score as compared with standard drugs used.

\subsubsection{Petra Calculations}

PETRA (Parameter Estimation for the Treatment of Reactivity Application)s is a program package comprising various empirical methods for the calculation of physico-chemical properties in organic molecules. All methods are empirical in nature and have been developed over the last 20 years in the research group of Prof. J. Gasteiger [29]. The following chemical effects can be quantified: heats of formation, bond dissociation energies, sigma charge distribution, $\pi$-charge distribution, inductive effect, resonance effect and delocalization energies and polarizability effect [30]. The compounds (4a) and (4b) have been subjected to delocalised-charge calculations using Petra method of the non-hydrogen common atoms (Figure.1), obtained from the partial $\pi$-charge of the heteroatoms, have been used to model the bioactivity against bacteria. It is found that the negative charges of the nitrogen atoms and the partial $\pi$ positive charges of oxygen atoms contribute positively in favour of an antibacterial activity, more, and this is in good agreement with the mode of antibacterial action of the compounds bearing (X $\delta--\mathrm{Y} \delta+$ ) pharmacophore(s) site(s). It was hypothesized that difference in charges between two heteroatoms of the same pharmacophore site $(\mathrm{X} \delta--\mathrm{Y} \delta+)$ may facilitate the inhibition of bacteria, more than viruses.

This hypothesis was rationalised as follows: The structure of synthesized coumarin derivatives (4a) and (4b) for ease of analysis can be divided into three parts, viz., coumarin skeleton, chromone moiety and another phenyl ring as side chain attached to methylene carbon by a Nitrogen i.e. the benzocaine. We have fixed the former coumarin skeleton and benzocaine part and varied the remaining one by chromone and quinoline moieties. Both the compounds (4a) and (4b) were found to be very active against a broad spectrum of bacterial strains. In case of compound (4a) there is comparable biological activity against S. aureus, Ps. aeruginosa, Klebsiella spp. and moderate activity against the remaining strains and in case of compound (4b) it was found highly active against S. aureus , S. pyogenes, Salmonella typhi and moderate against the remaining strains. Accordingly, an effort was initiated to establish a pharmacophore hypothesis to delineate the requirements of the active site via a comprehensive program of analogue synthesis and evaluation of the effects of structural modification(s) on antibacterial activity of $4 \mathrm{a}$ and $4 \mathrm{~b}$. We then set out to determine the resultant in vitro effects of chemical alterations in each region. The modulating antibacterial effect(s) of substituent having different electronegative properties, located at the $\mathrm{N}$ and two phenyl ring sites were ascertained next. A combination of the intact coumarin skeleton and the chromone and quinoline generated higher antibacterial activity (MICaround100) with respect to inhibition tests. We postulate that the strong tendency to form a $(\mathrm{N} \delta+-\mathrm{O} \delta-)$ dipolar pharmacophore site in the predominant form is likely to be responsible for the rise of biological activity observed with these semi $\pi$-conjugated derivatives. If this hypothesis is correct, by modifying both the compounds we may be able to modulate the degree of interaction of the compound with various bacteria.

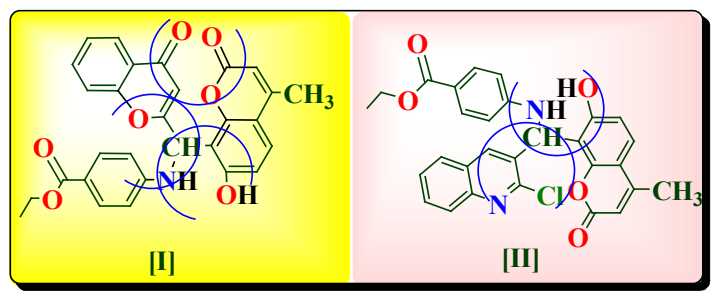

Figure 1. Potential antibacterial pharmacophores sites of the compounds (4a) and (4b)

\section{Conclusion}

Coumarin based Betti's condensed molecules containing chromone and quinoline moiety was synthesized in good yield utilizing the Betti's protocol. All the compounds were characterized on the basis of elemental and spectral data. The produced compounds were studied theoretically. Practically, all the molecules were screened against the panels of certain pathogenic strains of bacteria. Compounds $4 \mathrm{a}$ and $4 \mathrm{~b}$ were 
found to be equally potent comparable with amoxicillin and Streptomycin. Thus, from the data obtained from virtual and practical screening, it is concluded that the compounds were varied to possess a broad range of lipophilic character, revealed by $\log \mathrm{P}$ values. All the compounds were determined to express only one violation to the Rule of 5 , hence an indication of favorable bioavailability based on drug likeness. The considerable number of hydrogen donor/acceptor atoms incurred significant hydrophilic character into the majority of these drugs (supported by $\log \mathrm{P}$ values less than 5). Comparing relative activity scores of Ampicillin and Streptomycin utilizing four drug classes (GPCR ligand, ion channel modulator, kinase inhibitor, and nuclear receptor ligand) showed that all compounds are very highly correlated with expected similar bioactivity. On the basis of hypothesis based on Petra, it is found that, these compounds could form the highly interesting combined two or more pharmacophores sites in one molecule typically. It is predicted that most of these compounds could be used without great risk of toxicity in diverse antibacterial activity. Finally, going for the 3D dock studies revealed the structural modifications for rendering the enhancement in its biological activity. The modeling of the pharmacophores on the heterocyclic moieties i.e. the coumarin, chromone and the quinoline moieties can be done.

\section{REFERENCES}

[1] Irena Kostova, Curr. Med. Chem. - Anti-Cancer Agents, 5, (2005), 29-46.

[2] R. O'Kennedy, R. Douglas, Coumarins: Biology, Applications and Mode of Action. Wiley, New York, 1997.

[3] P. Wells, H. Morrison, Photo-cycloaddition of coumarin to tetramethylethylene. Photoreaction associated with the apparent interception of the coumarin singlet excimer. J. Am. Chem. Soc. 97 (1975) 154-159.

[4] D. Egan, R. O'Kennedy, E. Moran, D. Cox, The pharmacology, metabolism, analysis and applications of Coumarin and Coumarin related compounds. Drug Metab. Rev. 22 (1990) 503.

[5] H. Kitagawa, R. Iwaki, Coumarin derivatives for medicinal purposes. J. Pharm. Soc. Jpn. 83 (1963) 1124-1128.

[6] X. Pan et al. / Bioorg. Med. Chem. 14 (2006) 2771-2778

[7] V Srivastava et al., Bioinformation, 3 (2008) 180 [PMID:1923-8244].

[8] I. Kostova et al., Bioinorganic Chemistry and Applications. $1-9(2006)$

[9] Jonathan G. Heddle et al., Nucleosides, Nucleotides and Nucleic Acids. 19(8), (2000), 1249-1264.

[10] M. Betti, $\beta$-Napthol Phenylaminomethane. Org. Synth. 1 (1941) 381-383.

[11] M. Varughese, B. Ramkrishna, S. Shriniwas, "Bismuth(III) nitrate pentahydrate, a mild and inexpensive reagent for synthesis of coumarins under mild conditions"; Tetrahedron Lett. 46 (2005) 6957.

[12] A. A. Abu-Husse, Synthesis and spectroscopic studies on ternary bis-schiffbase complexes having oxygen and/or nitrogen donors. J. Coord. Chem. 59,(2006), 157-176.

[13] M. Sithambaram Karthikeyan, D. Jagadesh Prasad, B. Poojary, K. Subramanya Bhat, Synthesis and biological activity of Schiff and Mannich bases bearing2,4-dichloro-5-fluorophenyl moiety. Bioorg. Med. Chem. 14, (2006), 7482-7489.

[14] K. Singh, M.S. Barwa, P. Tyagi, Synthesis, characterization and biological studies of $\mathrm{Co}(\mathrm{II}), \mathrm{Ni}(\mathrm{II}), \mathrm{Cu}(\mathrm{II})$ and $\mathrm{Zn}(\mathrm{II})$ complexes with bidentate Schiff base derived from 4-amino-3-mercapto-6-methyl-5-oxo-1,2,4-triazine. Eur. J. Med. Chem. 41 (2006) 1-7.

[15] P. Pannerselvam, R.R. Nair, G. Vijayalakshmi, E.H. Subramanian, S.K. Sridhar, Synthesis of Schiff bases of 4-(4-aminophenyl)-morpholine as potential antimicrobial agents. Eur. J. Med. Chem. 40 (2005) 225-229.

[16] S.K. Sridhar, M. Saravan, A. Ramesh, Synthesis of Schiff bases of 4-(4-aminophenyl)- morpholine as potential antimicrobial agents. Eur. J. Med. Chem. 36 (2001) 615-625.

[17] M. Varughese, B. Ramkrishna, S. Shriniwas, Bismuth(III) nitrate pentahydrate a mild and inexpensive reagent for synthesis of coumarins under mild conditions. Tetrahedron Lett. 46 (2005) 6957.

[18] S.N. Pandeya, D. Sriram, G. Nath, E. Declercq, Synthesis, antibacterial, antifungal and anti-HIV activities of Schiff and Mannich bases derived from isatin derivatives and N-[4-(40-chlorophenyl) thiazol-2-yl] thiosemicarbazide. Eur. J. Pharmacol. 9 (1999) 25-31.

[19] R. Mladenova, M. Ignatova, N. Manolova, T. Petrova, I. Rashkov, Preparation, characterization and biological activity of Schiff base compounds derived from 8-hydroxyquinoline-2-carboxaldehyde and Jeffamines. Eur. Polym. J. 38 (2002) 989-998. activity. Eur. J. Med. Chem. 31 (1996) 989-1000.

[20] M. Ghandi, A. Olyaei, S. Raoufmoghaddam; Synthetic Communications, 38 (2008), 4125-4138.

[21] A. Parvez, M. Jyotsna, H. Taibi Ben, "Theoretical prediction and experimental verification of antibacterial potential of some monocyclic $\beta$-lactams containing two synergetic buried antibacterial pharmacophore sites"; Phosphorus, Sulphur Silicon Relat. Elem. 185 (2010) 1-11.

[22] Online Available: http://www.molinspiration.com

[23] P. Ertl, B. Rohde, P. Selzer, Fast calculation of molecular Polar Surface Area (PSA) as a sum of fragment-based contributions and its application to the prediction of drug transport properties. J. Med. Chem. 43 (2000) 3714-3717.

[24] L.C.W. Chang, R.F. Spanjersberg, M.W. Beukers, A.P. IJzerman, 2, 4,6-Trisubstituted pyrimidines as a new class of selective adenosine A-1 receptor antagonists. J. Med. Chem. 47 (2004) 6529-6540.

[25] D. E. Clark, Rapid calculation of polar molecular surface area and its application to the prediction of transport phenomena. J. Pharm. Sci. 88 (1999) 807-814. 
[26] V.N. Viswanadhan, A.K. Ghose, G.R. Revankar, R.K. Robins, Atomic physicochemical parameters for three dimensional structure directed quantitative structure activity relationship: additional parameters for hydrophobic and dispersive interaction and their application for an automated superposition of certain naturally occurring nucleoside antibiotics. J. Chem. Inf. Comput. Sci. 29 (1989) 163-172.

[27] C.A. Lipinski, F. Lombardo, B.W. Dominy, P.J. Feeney, Experimental and computational approaches to estimate solubility and permeability in drug discovery and development settings. Adv. Drug Deliv. Rev. 46 (2001) 3-26.

[28] Online available from http://www.osiris.com

[29] Petra molecular properties calculations program's is freely available online at the following site: http://www2.chemie-unierlangen.de/services/petra

[30] Morris GM, Goodsell DS, Halliday RS, Huey R, Hart WE, Belew RK, et al. Automated docking using a Lamarckian genetic algorithm and empirical binding free energy function. J Comput Chem 19: (1998)1639-62 